\title{
АВТОМАТИЗАЦИЯ ЛИТЬЕВЫХ МАШИН НА БАЗЕ ПРОГРАММИРУЕМЫХ ЛОГИЧЕСКИХ КОНТРОЛЛЕРОВ
}

\section{AUTOMATION OF CASTING MACHINES BASED ON PROGRAMMABLE LOGIC CONTROLLERS}

\author{
M. Rusakov \\ A. Evdokimov \\ A. Zankin
}

Summary. Postgraduate student, Federal State Budgetary Educational Institution of Higher Education N.P. Ogarev National Research Mordovian State University, Saransk

The article discusses the practical application of programmable logic controllers of the "Aries" company for the automation of a single-position machine for injection molding of thermoplastic materials LPD500/160. The article briefly discusses the solutions to the main problems of automation of injection molding machines: the choice of equipment and the creation of a user program. This article is written on a project that has a practical implementation in production when performing a major overhaul of equipment.

The purpose of this work is to show the main ways of solving the problems of automation of injection molding machines of this class.

Keywords: PLC, mold, cycle, automation, subroutine, injection unit, clamping unit, interfaces, injection molding machine, hydraulic valve, command device.
Русаков Максим Геннадьевич

Aсnирант, ФГБОУВО «Национальный исследовательский Мордовский государственный университет имени Н.П. Огарёва», Саранск e10e00@mail.ru

Евдокимов Александр Сергеевич Аспирант, ФГБОУВО «Национальный исследовательский Мордовский государственный университет имени Н.П. Огарёва», Саранск evd15@list.ru

Занкин Александр Иванович

Аспирант, ФГБОУ ВО «Национальный исследовательский Мордовский государственный университет имени Н.П. Огарёва», Саранск zankin_ai@mail.ru

Аннотация. В статье рассматривается практическое применение программируемых логических контроллеров фирмы «Овен» для автоматизации однопозиционной машины для литья под давлением термопластичных материалов ЛПД 500/160. В статье кратко рассматривается решения основных проблем автоматизации литьевых машин: выбор оборудования и создания пользовательской программы. Данная статья написана по проекту имеющие практическое внедрение на производстве при выполнении капитального ремонта оборудования.

Цель данной работы — показать основные пути решения проблем автоматизации литьевых машин данного класса.

Ключевые слова: ПЛК, прессформа, цикл, автоматизация, подпрограмма, узел впрыска, узел смыкания, интерфейсы, литьевая машина, гидрораспределитель, командоаппарат.

мощностях поставила задачу по восстановлению работоспособности данного оборудования. Установка нового дорогого импортного оборудования АСУ ТП на такие машины была бы не рентабельной. Поэтому было принято решения использовать продукцию отечественного производителя. Проблема выбора оборудования для автоматизации литьевых машин решалась следующим образом. На начальных этапах проводится изучение оригинальной документации, изучается работа литьевой машины и анализируется рынок современного оборудования АСУ ТП. Далее проводится выбор того или иного подходящего оборудования для автоматизации.

Общая теоретическая часть выбора оборудования АСУ ТП т.е. критерии его оценки приводится на рисунке 1. Только взвесив и сопоставив правильно все критерии 


\section{КРИТЕРИЙ ОЦЕНКИ}

\section{Технические характеристки}

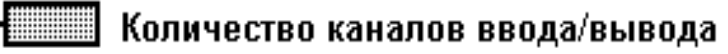

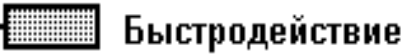

世:? Уровни напряжений входов/выходов

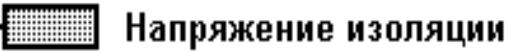

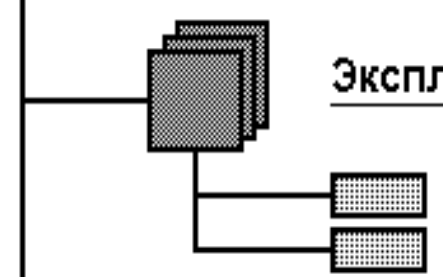

Диапазон рабочих тенператур

Относительная влажность воздуха
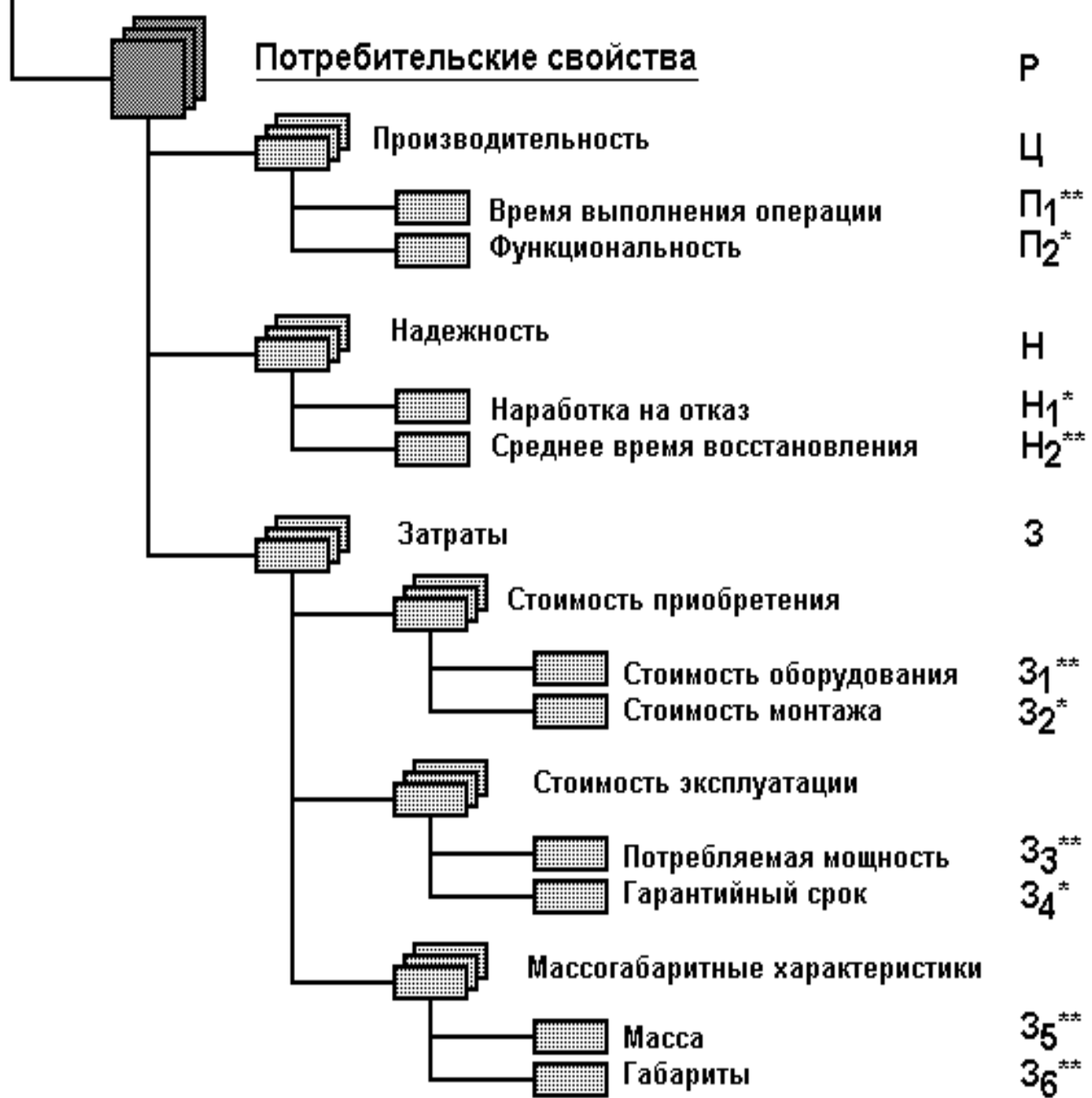


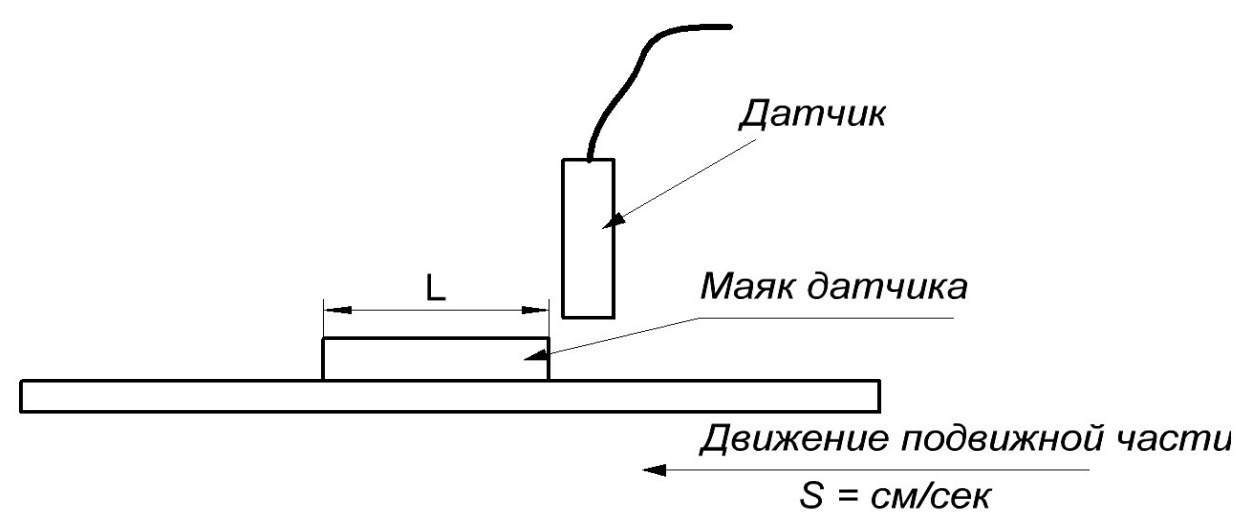

Рис. 2. К определению времени импульса с датчика узла запирания

выбора оборудования можно сделать правильный выбор. На верхнем уровне дерева критериев располагаются технические характеристики они являются одними из важных без них невозможно провести автоматизацию оборудования.

Для определения технических характеристик используемого оборудования проводится изучение электрических принципиальных схем, подсчитываются количество используемых цифровых и аналоговых входов и выходов, определяются величины входных и выходных сигналов.

В результате проведенных исследований для автоматизации литьевой машины потребовалось использовать АСУ ТП имеющую на борту 39 дискретных входа, 24 дискретных выхода и 4 аналоговых входа для подключения термопар. Уровни входных цифровых сигналов должны соответствовать напряжению 24В постоянного тока. Уровни выходных цифровых сигналов должны соответствовать 24В постоянного тока величиной тока не менее 2 ампер. Уровни входных аналоговых сигналов должны соответствовать уровням термопар хромель-копель. Для связи ПЛК и человеко-машинного интерфейса необходима цифровая сеть.

Другой важной технической характеристикой для автоматизации литьевой машины ЛПД 500/160 является минимально необходимое максимально допустимое время реакции АСУ ТП на входной сигнал. Для выполнения этих требований необходимо чтобы ПЛК системы управления имело минимально допустимое время цикла или имело возможность выполнения программы прерываний. Для работы литьевой машины ЛПД 500/160 время реакции опирается на скорость движении механизма запирания прессформы (рисунок 2). В силу своих конструктивных и технологических особенностей на однопозиционной литьевой машине ЛПД 500/160 макси- мальная скорость может находится только на двух механизмах: механизм впрыска и механизм узла запирания. Наиболее важным является узел запирания поскольку он имеет самые короткие импульсы, на которые системе необходимо успеть отреагировать.

При известной максимальной скорости перемещения прессформы $0.8 \mathrm{~m} / \mathrm{c}$ и длине пластины бесконтактного выключателя 2,5см, время импульса с датчика составит 31мс. В течение этого времени датчик должен быть опрошен и система управления должна сформировать управляющий сигнал. В противном случае остановка прессформы произойдёт не на пластине датчика, а за его пределами или вовсе не произойдёт.

Не менее важными характеристиками так же являются потребительские свойства и эксплуатационные характеристики. Для внутрицеховых условий со средней возможной температурой эксплуатации от 5 до 25区 и перечисленными выше требованиями наиболее подходящими являются оборудование производящиеся фирмой «Овен», оно достаточно надежное и относится к невысокой ценовой категории. За основу системы управления литьевой машины ЛПД 500/160 был принят моноблочный контроллер ПЛК110-24.60.Р.М на 60 точек подключения. Недостаточность цифровых и аналоговых входов на борту ПЛК компенсируется применением дополнительных модулей расширения. В таблице 1 и 2 приведено все используемое оборудование АСУ ТП для автоматизации литьевой машины. Для сопряжения ПЛК и модулей расширения задействованы интерфейсы RS485-1 и RS485-2 имеющиеся на борту ПЛК. В качестве основного протокола связи используется протокол ModBus-RTU. На рисунке 3 приведена структурная схема конфигурации проекта.

Так как литьевая машина ЛПД-500/160 является гидравлической литьевой машиной, все управления ра- 
Таблица 1. Таблица конфигурации оборудования АСУ в сети Modbas по интерфейсу RS485-1

\begin{tabular}{|l|l|l|}
\hline № & Единица оборудования АСУ в сети & Адрес в сети Моdbas \\
\hline 1 & ПЛК110-24.60.Р.М (мастер устройство) & \\
\hline 2 & МВ110-24.16ДН & 16 \\
\hline 3 & МВ110-8А & 24 \\
\hline
\end{tabular}

Таблица 2. Таблица конфигурации оборудования АСУ в сети Modbas по интерфейсу RS485-2

\begin{tabular}{|l|l|l|}
\hline № & Единица оборудования АСУ в сети & Адрес в сети Моdbas \\
\hline 1 & ИПЗ20 (мастер устройство) & \\
\hline 2 & ПЛК110-24.60.Р.М & 1 \\
\hline
\end{tabular}

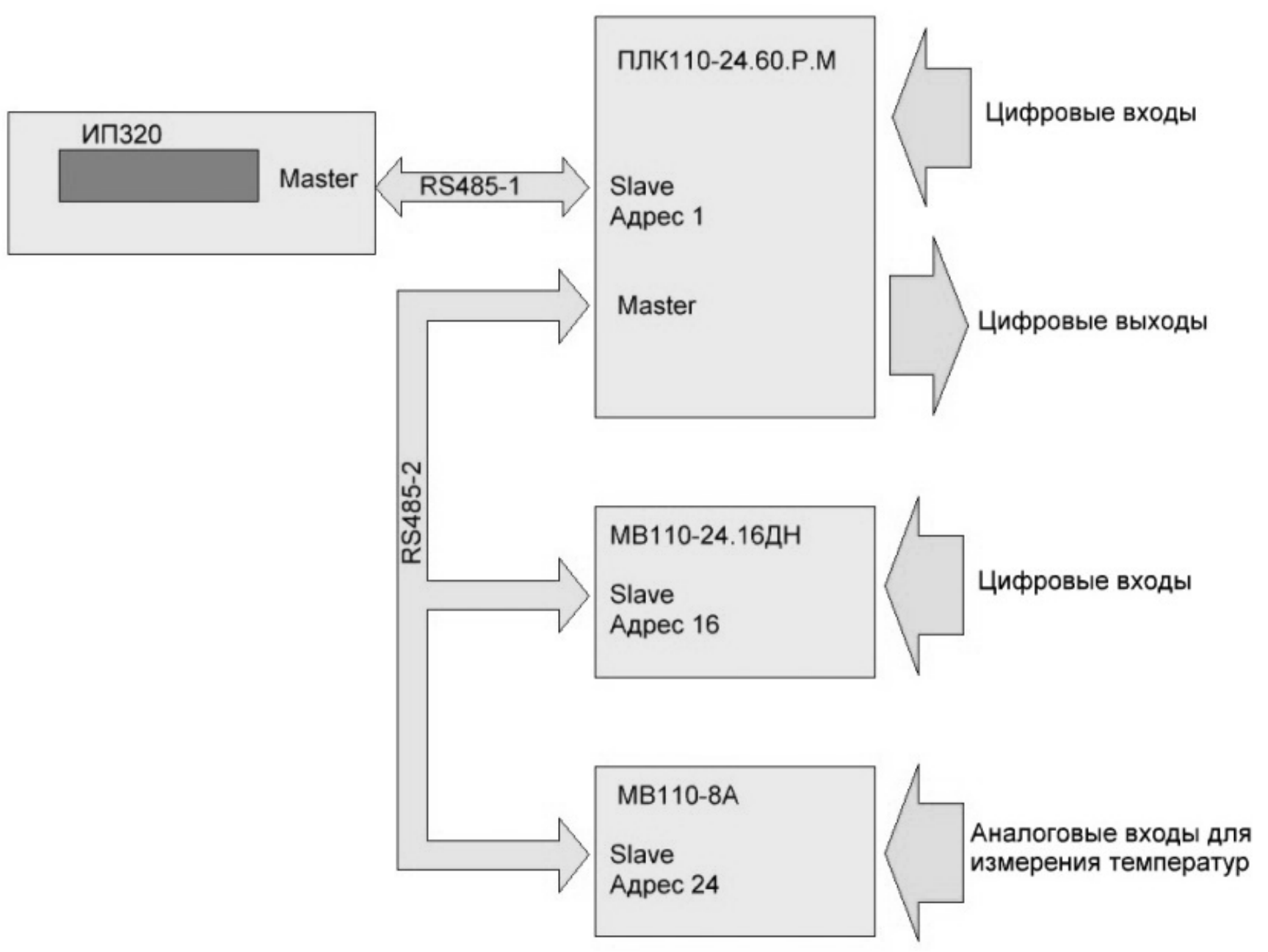

Рис. 3. Структурная схема АСУ ТП литьевой машины ЛПД 500/160 


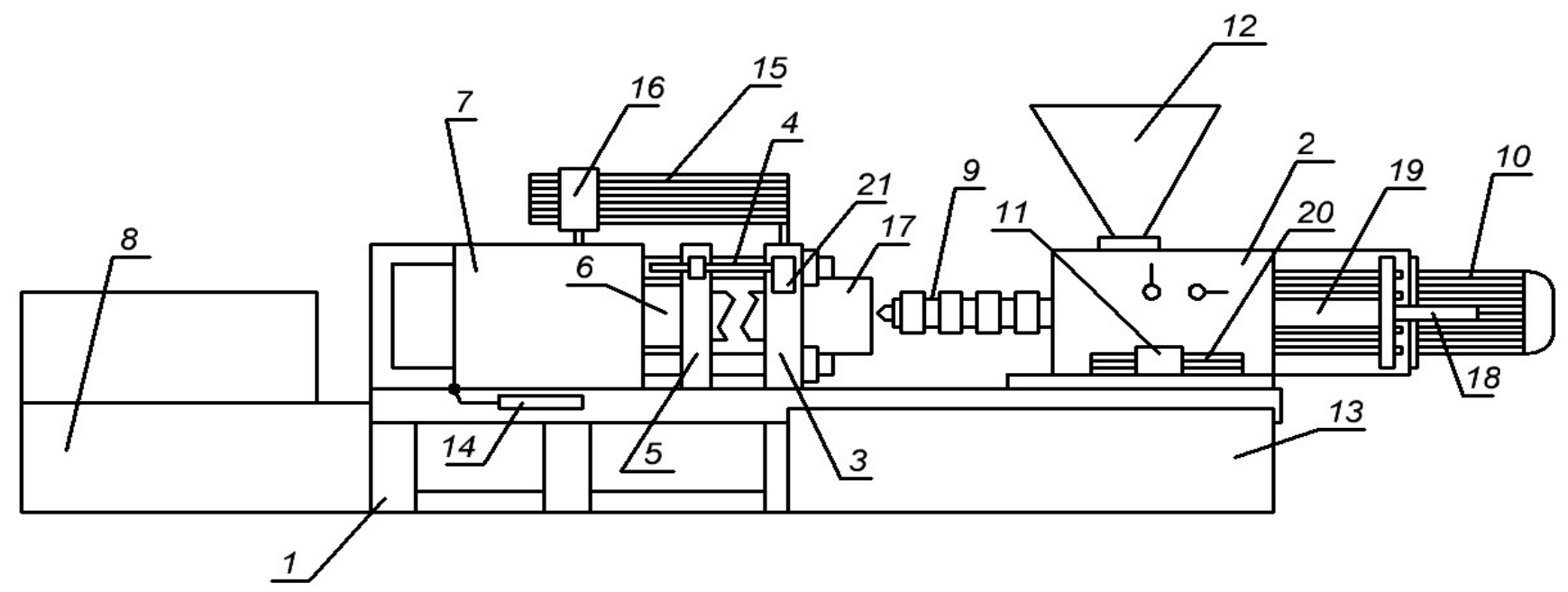

Рис. 4. Общий вид литьевой машины ЛПД-500/160

1 - станина, 2 - редуктор узла впрыска, 3 - неподвижная плита узла смыкания, 4 - направляющие колонки узла смыкания, 5 - подвижная плита узла смыкания, 6 - главный гидроцилиндр подвижной плиты, 7 - ограждения пространства прессформы, 8 - гидростанция, 9 - цилиндр пластикации, 10 - электродвигатель шнека цилиндра пластикации, 11 - блок датчиков положения узла впрыска, 12 - загрузочный бункер, 13 - шкаф управления, 14 - блок датчиков ограждения прессформы, 15 - рейка установки маяков для датчиков подвижной плиты, 16 - блок датчиков подвижной плиты, 17 - ограждение узла впрыска, 18 - шток гидроцилиндра впрыска с датчиком положения, 19 гидроцилиндр впрыска, 20 - рейка установки маяков для датчиков положения узла впрыска, 21 блок датчиков высоты прессформы и высокого давления.

бочими механизмами машины осуществляются за счёт включения соответствующих клапанов на гидрораспределителях. Положение рабочих механизмов машины определяется срабатыванием соответствующих датчиков на командоаппаратах, расположенных по периферии машины, которые являются частью системы управления.

Промышленное оборудование подобного типа имеет примерно одинаковое устройство. В своем составе они содержат следующие основные узлы: несущую раму, узел смыкания прессформы, узел впрыска и гидростанцию. На рисунке 4 схематично представлен более подробно общий вид машины ЛПД 500/160 с пояснениями деталей машины.

Работа ЛПД 500/160 осуществляется в четырех режимах: наладочный режим, ручной режим, полуавтоматический режим и автоматический режим. Каждый режим работы имеет свои отличительные особенности поэтому программа пользователя для ПЛК110-24.60.Р.М содержит подпрограммы этих режимов работы, которые вызываются в главную программу для циклической обработки.

Наладочный режим используется в момент установки прессформы и наладки производства нового изделия.
В этом режиме все рабочие органы машины совершают движения только с одной скоростью и только в момент нажатия кнопок на пульте оператора. Движение рабочих органов прекращаются, когда кнопка отпущена или когда сработает соответствующий датчик ограничения.

Режим ручного прессования запускает в работу каждый механизм машины с соответствующей кнопки пульта оператора и с поддержкой давления в рабочем механизме на протяжении полного цикла литья. Данный режим позволяет запускать технологический процесс литья под давлением поэтапно после нажатия соответствующих кнопок на пульте оператора. Такой режим используется во время отладки нового изделия или подготовки к автоматическому или полуавтоматическому режиму литья. Для запуска ручного режима необходимым разрешающим сигналом является маркер прогретого цилиндра пластикации, наличия сигналов: закрытия ограждения узла впрыска, ограждение пространства прессформы, толкатель убран.

Полуавтоматический режим характеризуется запуском одного полного цикла литья под давлением либо с кнопки смыкания прессформы или импульсом с датчика закрытия ограждения прессформы. В данном режиме имеет значение исходное состояние машины. Исходное состояние машины подготавливается в ручном режиме. 


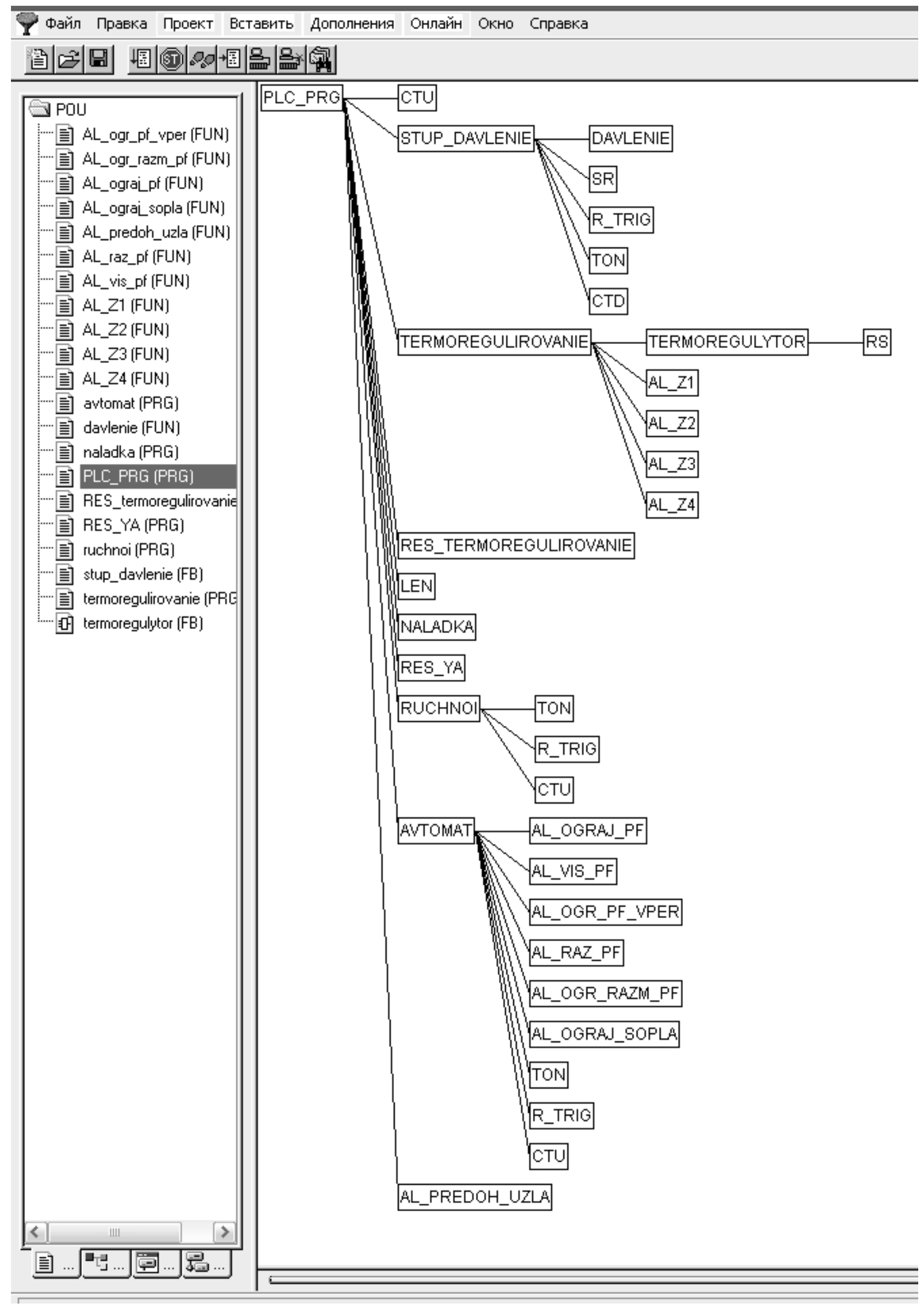

Рис. 5. Рабочее окно проекта с деревом вызовов 


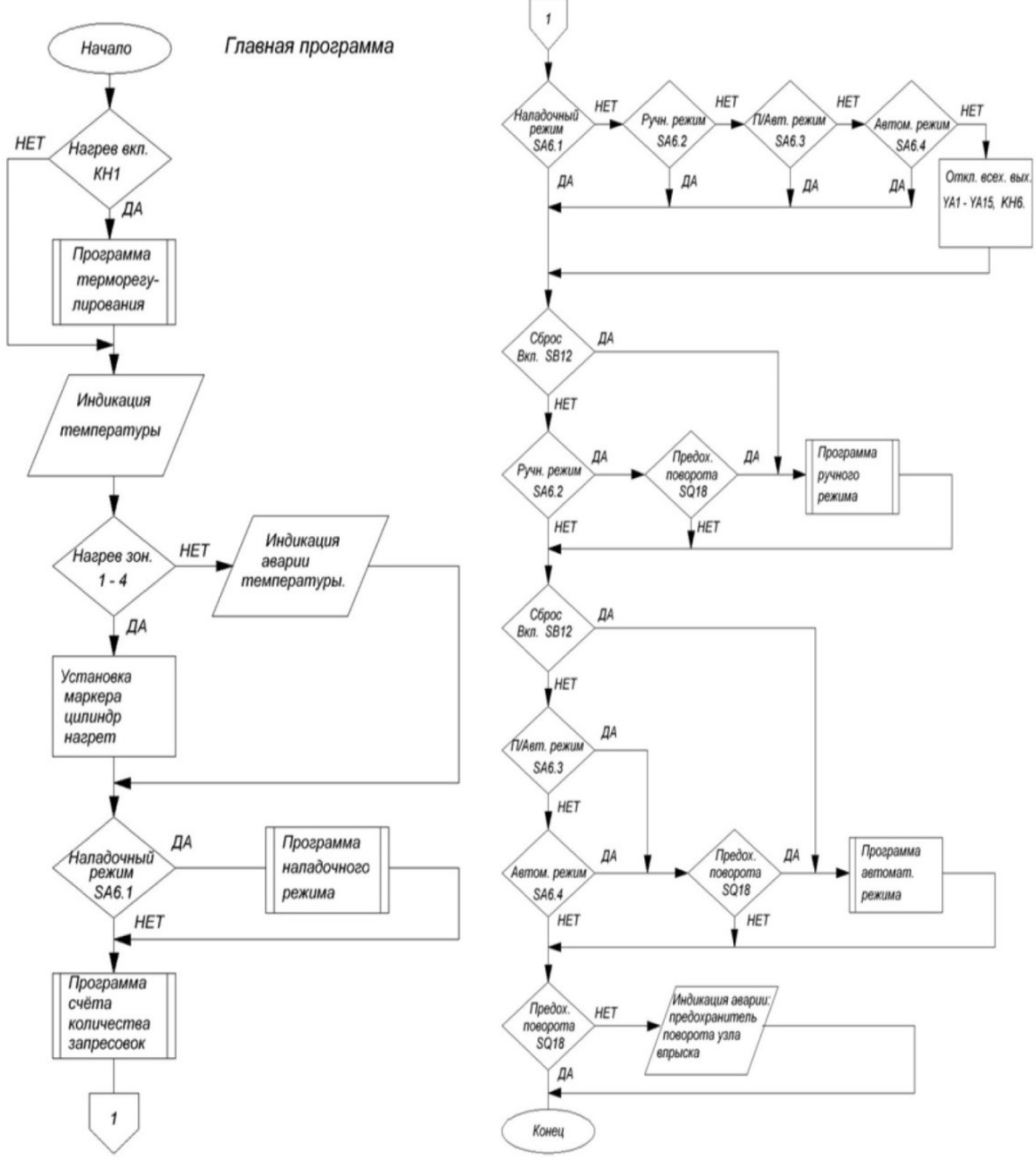

Рис. 6. Алгоритм работы главной программы 
Разрешающими сигналами для запуска полуавтоматического и автоматического режимов служат, сигналя с датчиков: прессформа раскрыта (подвижная плита находится в исходном положении), материал набран в цилиндре пластикации (шток гидроцилиндра выдвинут), ограждение узла впрыска закрыто, ограждение пространства прессформы закрыто, толкатель убран, цилиндр пластикации прогрет. В конце цикла литья после экстракции изделия из прессформы толкатель убирается, и машина приходит в режим ожидания новой команды запуска литья.

Автоматический режим работы отличается от полуавтоматического тем что машина работает непрерывно по таймеру, не ожидая команд от оператора.

Для обеспечения всех режимов работы литьевой машины на рисунке 5 представлена структура программы ПЛК110-24.60.Р.М в среде Codesys v2.3.

Как видно из рисунка программа в своем составе содержит 21 программный элемент - это функции, функциональные блоки и подпрограммы, вызываемые в главную программу при различных условиях.

На начальных этапах написания программы, в соответствии с технологией литья, составляется алгоритм работы станка. На рисунке 6 приведен алгоритм работы главной программы. На диаграмме изображены вызовы подпрограмм: терморегулирования, наладочного режима работы, ручного режима работы, программа подсчёта количества запрессовок, автоматического режима работы. В программе пользователя отсутствует как самостоятельный элемент подпрограмма полуавтоматического режима прессования, его функции выполняет подпрограмма автоматического режима работы с применением условий полуавтоматического режима. Программа терморегулирования узла пластикации обеспечивает терморегулирование четырех зон нагрева с применением метода двухпозиционного регулирования. Для этого в функциональный блок «TERMOREGULYTOR» вызываются соответствующие экземпляры (рисунок 5).

Помимо всего того что обеспечивает технологию процесса литья в программе предусмотрены блокировки обеспечивающие безопасную работу оператора.

Часть кода работы главной программы приведена ниже.

\section{АИСТИНГ \\ г^авной программы}

PROGRAM PLC_PRG

VAR stup_davlen: stup_davlenie; (* объявление экземпляра функционального блока выдержки под давлением*)

schet_zapresovok: CTU; (*библиотечный функциональный блок- суммирующий счётчик*)

END_VAR

(*ИНИЦИАЛИЗАЦИЯ ГЛОБАЛЬНЫХ ПЕРЕМЕННЫХ ДОПОЛНИТЕЛЬНОГО МОДУЛЯ ВВОДА МВ110-16ДН*)

ograj_pf_sq:=m_in.0; (*Побитная распиновка регистра 51 (регистра входов)*)

ogr_skor_pf_sq:=m_in.1;

uvel_skor_pf_sq:=m_in.2;

zamed_skor_pf_sq:=m_in.3;

ogr_pf_vper_sq:=m_in.4;

vis_dav_res_sq:=m_in.5;

ogr_razm_pf_sq:=m_in.6;

vis_dav_sq:=m_in.7;

predohran_sq:=m_in.8;

podscok_sq:=m_in.9;

vis_pf_sq:=m_in.10;

kon_vprisk_sq:=m_in.11;

kon_nabora_sq:=m_in.12;

ogr_pod_sq:=m_in.13;

ogr_otvod_sq:=m_in.14;

avar_otvod_sq:=m_in.15;

(*ИНИЦИАЛИЗАЦИЯ ГЛОБАЛЬНОЙ ПЕРЕМЕННОЙ ПРИХОДЯЩЕЙ С ПАНЕЛИ ОПЕРАТОРА*)

R_schet_zap:=in 1.4; ( ${ }^{*}$ сброс счётчика запресовок*)

(*ОТРАБОТКА ЭНЕРГОНЕЗАВИСИМЫХ ПЕРЕМЕННЫХ*) (*ЗАПИСЬ В ЭНЕРГОНЕЗАВИСИМУЮ ПАМЯТЬ ИЗ РЕГИСТРОВ ПАНЕЛИ ОПЕРАТОРА $*$

UZ1:=UZ1_;
UZ2:=UZ2_;
UZ3:=UZ3_;
UZ4:=UZ4_;
DZ1:=DZ1_;
DZ2:=DZ2_;
DZ3:=DZ3_;
DZ4:=DZ4_;

A_HI_Z1:=A_HI_Z1_;

A_HI_Z2:=A_HI_Z2_;

A_HI_Z3:=A_HI_Z3_;

A_HI_Z4:=A_HI_Z4_;

A_LO_Z1:=A_LO_Z1_;

A_LO_Z2:=A_LO_Z2_;

A_LO_Z3:=A_LO_Z3_;

A_LO_Z4:=A_LO_Z4_;

T1_Ust:=T1_Ust_;

T2_Ust:=T2_Ust_;

T3_Ust:=T3_Ust_;

T4_Ust:=T4_Ust_;

T5_Ust:=T5_Ust_;

kontrol_cikla_Ust:=kontrol_cikla_Ust_; 
Time_davlen:=Time_davlen_;

Ust_N_Stup:=Ust_N_Stup_;

Ust_schet_ogr_razm:=Ust_schet_ogr_razm_;

\section{(* РАБОТА С ТЕРМОРЕГУЛЯТОРОМ*)}

IF ON_temp (*если нагрев включен то вызывается программа termoregulirovanie*)

THEN termoregulirovanie; (* программа терморегулирования*)

ELSE RES_termoregulirovanie; (* в противном случае происходит сброс всех выходов с терморегулирования*)

END_IF (*через вызов программы сброса*)

(*ПЕРЕДАЧА ДАННЫХ ТЕМПЕРАТУРЫ НА ПАНЕЛЬ ОПЕРАТОРА ИПЗ20*)

Z1_:=Z1; (*1 ЗОНА НАГРЕВА С МОДУЛЯ ВВОДА НАПАНЕЛЬ ОПЕРАТОРА*)

Z2_:=Z2; (*2 3ОНА НАГРЕВА С МОДУЛЯ ВВОДА НАПАНЕЛЬ ОПЕРАТОРА $*$

Z3_:=Z3; (*3 3ОНА НАГРЕВА С МОДУЛЯ ВВОДА НАПАНЕЛЬ ОПЕРАТОРА $\left.{ }^{*}\right)$

Z4_:=Z4; (*4 3ОНА НАГРЕВА С МОДУЛЯ ВВОДА НАПАНЕЛЬ ОПЕРАТОРА*)

(*ПЕРЕДАЧА ДАННЫХ ВРЕМЕНИ НА ПАНЕЛЬ ОПЕРАТОРА ИПЗ20(передается целое + 1знак после запятой ${ }^{*}$ )

T1_tekuch_:=TRUNC( ( TIME_TO_REAL ( T 1 _ tekuch) $\left.)^{*} 0.01\right) ;\left({ }^{*}\right.$ время предохранение п. $\left.\phi^{*}\right)$

T2_tekuch $:=$ TRUNC( (TIME_TO_REAL (T2 tekuch) $\left.)^{*} 0.01\right) ;\left({ }^{*}\right.$ время декомпрессии*)

T3_tekuch_:=TRUNC((TIME_TO_REAL(T3_ tekuch) )*0.01);(*время впрыска*)

T4_tekuch _: =TRUNC( (TIME_TO_REAL ( T4_ tekuch))*0.01);(*время набора (пластикации) *)

T5_tekuch $:=$ TRUNC( (TIME_TO_REAL (T5 tekuch) $\left.)^{*} 0.01\right) ;\left({ }^{*}\right.$ время охлаждение $\left.{ }^{*}\right)$

tekuch_davlen_:= TRUNC((TIME_TO_REAL(tekuch_ davlen) $\left.{ }^{*} 0.01\right) ;\left({ }^{*}\right.$ время выдержки под давлением *)

kontrol_cikla_tekuch_:=TRUNC((TIME_TO_ REAL(kontrol_cikla_tekuch))*0.01);(*время контроля цикла*)

(*ПЕРЕДАЧА КОДОВ АВАРИЙНЫХ СИГНАЛОВ НА ПАНЕЛЬ ОПЕРАТОРА ИПЗ2О ДЛЯ ФОРМИРОВАНИЯ ДИНАМИЧЕСКОГО ТЕКСТА*)

AZ1_:= (SHR((LEN(AZ1)),3));(*определяем длину строковой переменной и уменьшаем величину полученного выражения*)

AZ2_:= (SHR((LEN(AZ2)),3)); (* получаем: «низкая температура зон нагрева» $=3$,» перегрев зон» $=2$, « обрыв термопары» $=4 *)$ *)

AZ3_:=(SHR((LEN(AZ3)),3)); (* «отсутствие аварии» $=0$

AZ4_:=(SHR((LEN(AZ4)),3));

(*выражение принимает значение 1, при наличии аварии и 0 при её отсутствии*)
A_CIKL_:=(SHR((LEN(A_CIKL)),4)); (*BЫВОД НА ПАНЕЛЬ ОПЕРАТОРА КОДА АВАРИЙНОГО СИГНАЛА - ВРЕМЯ ЦИКЛА*

A_PRED_:=(SHR((LEN(A_PRED)),5)); (*ВЫВОД НА ПАНЕЛЬ ОПЕРАТОРА КОДА АВАРИЙНОГО СИГНАЛА - ПРЕДОХРАНЕНИЯ ПРЕССФОРМЫ *)

A_VPRISK_:=(SHR((LEN(A_VPRISK)),4)); (*BЫВОД НА ПАНЕЛЬ ОПЕРАТОРА КОДА АВАРИЙНОГО СИГНАЛА - ВРЕМЯ ВПРЫСКА*)

A_NABOR_: $\left(\mathrm{SHR}\left(\left(\mathrm{LEN}\left(\mathrm{A} \_\mathrm{NABOR}\right)\right), 4\right)\right) ; \quad\left({ }^{*} \mathrm{~B}\right.$ ПАНЕЛЬ ОПЕРАТОРА КОДА АВАРИЙНОГО СИГНАЛА ВРЕМЯ НАБОРА*)

AL_ograjdenie_pf_:=(SHR((LEN(AL_ograjdenie_pf $)), 5))$; (* ВЫВОД НА ПАНЕЛЬ ОПЕРАТОРА КОДА АВАРИЙНОГО СИГНАЛА - ОГРАЖДЕНИЕ ПРЕССФОРМЫ *)

AL_visota_pf_:=(SHR((LEN(AL_visota_pf $)), 4))$; (* ВЫВОД НА ПАНЕЛЬ ОПЕРАТОРА КОДА АВАРИЙНОГО СИГНАЛА ВЫСОТА ПРЕССФОРМЫ*)

$A L_{-}$ogranichenie_pf_vp_: $\left(\mathrm{SHR}_{-}\left(\left(\mathrm{LEN}\left(\mathrm{AL} \mathrm{L}_{-}\right.\right.\right.\right.$ ogranichenie_pf_vp)),5));(* ВЫВОД НА ПАНЕЛЬ ОПЕРАТОРА КОДА АВАРИЙНОГО СИГНАЛА - ОГРАНИЧЕНИЕ СМЫКАНИЯ ПРЕССФОРМЫ*)

AL_razmikanie_:=(SHR((LEN $\left.\left.\left.\left(A L \_r a z m i k a n i e\right)\right), 4\right)\right) ;\left(^{*}\right.$ BbВОД НА ПАНЕЛЬ ОПЕРАТОРА КОДА АВАРИЙНОГО СИГНАЛА - РАЗМЫКАНИЕ ПРЕССФОРМЫ ОПЕРАТОРОМ $\left.{ }^{*}\right)$

AL_polojenie_pf_:=(SHR((LEN $\left.\left.\left.\left(A L \_p o l o j e n i e \_p f\right)\right), 5\right)\right) ; \quad(*$ ВЫВОД НА ПАНЕЛЬ ОПЕРАТОРА КОДА АВАРИЙНОГО СИГНАЛА — НАЧАЛЬНОЕ ПОЛОЖЕНИЕ ПРЕССФОРМЫ

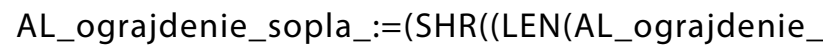
sopla)),4));(*ВЫВОД НА ПАНЕЛЬ ОПЕРАРОРА КОДА АВАРИЙНОГО СИГНАЛА — ОГРАЖДЕНИЕ СОПЛА НЕ ЗАКРЫ$\left.\mathrm{TO}^{*}\right)$

$A L_{-}$predoh_povorota_:=(SHR((LEN $\left(A L_{-}\right.$predoh_ povorota)),4)); (*ВЫВОД НА ПАНЕЛЬ ОПЕРАРОРА КОДА АВАРИЙНОГО СИГНАЛА - ПРЕДОХРАНИТЕЛЬ ПОВОРОТА УЗЛА ВПРЫСКА *)

(*ПЕРЕДАЧА СИГНАЛОВ С ДИСКРЕТНЫХ ДАТЧИКОВ НА ПАНЕЛЬ ОПЕРАТОРА*)

out1.0:=vis_dav_res_sq; (*КОНЦЕВИК СБРОСА ВЫСОКОГО ДАВЛЕНИЯ*)

out1.1:=vis_dav_sq; (*КОНЦЕВИК ВКЛЮЧЕНИЯ ВЫСОКОГО ДАВЛЕНИЯ*)

out1.2:=ogr_pf_vper_sq; (*КОНЦЕВИК ОГРАНИЧЕНИЕ ПРЕССФОРМЫ ВПЕРЕД*)

out 1.3:=ograj_pf_sq; ( ПРЕССФОРМЫ ЗАКРЫТО*)

out1.4:=vis_pf_sq; (*КОНЦЕВИК ВЫСОТА ПРЕССФОР$\mathrm{Mbl}^{*}$ )

out 1.5:= ogr_razm_pf_sq; (* КОНЦЕВИК ОГРАНИЧЕНИЕ РАЗМЫКАНИЯ ПРЕССФОРМЫ $\left.{ }^{*}\right)$

out 1.6:= ogr_skor_pf_sq; ( ${ }^{*}$ КОНЦЕВИК ОГРАНИЧЕНИЕ СКОРОСТИ ПРЕССФОРМЫ ПРИ СМЫКАНИИ*)

out1.7:=ogr_pod_sq; $\quad\left({ }^{*}\right.$ КОНЦЕВИК ОГРАНИЧЕНИЕ ПОДВОДА СОПЛА*) 
out1.8:=ogr_otvod_sq; (* КОНЦЕВИК ОГРАНИЧЕНИЕ ОТВОДА СОПЛА*)

out1.9:=avar_otvod_sq; ( ${ }^{*}$ АВАРИЙНОЕ ОГРАНИЧЕНИЕ ОТВОДА СОПЛА*)

out1.10:=kon_vprisk_sq; $\left({ }^{*}\right.$ КОНЦЕВИК ОКОНЧАНИЕ ВПРЫСКА*) $\mathrm{PA}^{*}$ )

out1.11:=kon_nabora_sq; (*КОНЦЕВИК КОНЕЦ НАБО-

out1.12:=podscok_sq;(* КОНЦЕВИК ОСТАНОВКА П.Ф ПРИ ПОДСКОКЕ ВПЕРЕД*)

out 1.13:= predohran_sq;(*КОНЦЕВИК НАЧАЛО ПРЕДО-

ХРАНЕНИЯ П. $\left.\Phi^{*}\right)$

out1.14:= uvel_skor_pf_sq; (* УВЕЛИЧЕНИЕ СКОРОСТИ

ПРИ РАЗМЫКАНИИ ПРЕССФОРМЫ

out1.15:= zamed_skor_pf_sq;(* УMEHЬШЕНИЕ CKOPO-

СТИ ПРИ РАЗМЫКАНИИ ПРЕССФОРМЫ $\left.{ }^{*}\right)$

out2.0:= predoh_povorota_sq; ${ }^{*}$ ПРЕДОХРАНИТЕЛЬ ПОВОРОТА УЗЛА ВПРЫСКА*)

out2.1:=ograj_s_zak_sq; (*ОГРАЖДЕНИЕ СОПЛА ЗАKPЫTO *)

out2.2:=davl_sopla_sq;(* ДАТЧИК ДАВЛЕНИЯ ПРИЖАТИЯ СОПЛА*)

out2.3:=OK_temp; (*ЦИЛИНДР ПЛАСТИКАЦИИ НАГPET*)

out2.4:=AVARIY; $\left({ }^{*}\right.$ АВАРИЙНЫЙ СИГНАЛ НА ПАНЕЛИ OПЕРАТОРА*)

(*ВЫЗОВ ПРОГРАММЫ НАЛАДОЧНОГО РЕЖИМА*)

IF naladka_sa (*если включен переключатель: наладочный режим*)

THEN naladka; (*то вызывается программа наладочного режима naladka*)

END_IF

( ${ }^{*}$ СБРОС ВСЕХ ВЫХОДОВ $\left.{ }^{*}\right)$

IF NOT (naladka_sa OR ruchnoi_sa OR avtomat_sa OR pol_avtomat_sa)(*если не включен не один режим*)

THEN RES_YA; ( ${ }^{*}$ сброс всех выходов *)

END_IF

\section{(*ВЫЗОВ ПРОГРАММЫ РУЧНОГО РЕЖИМА*)}

IF (ruchnoi_sa AND predoh_povorota_sq) OR reset_sb (*если включен переключатель: ручной режим или нажата кнопка сброс*)

THEN ruchnoi; (*то вызывается программа ручного режима либо для работы, либо для сброса параметров в ручном режиме*)

END_IF

(*ВЫЗОВ ПРОГРАММЫ АВТОМАТИЧЕСКОГО ИЛИ ПОЛУАВТОМАТИЧЕСКОГО РЕЖИМА*)

IF((avtomat_sa OR pol_avtomat_sa) AND predoh_ povorota_sq) OR reset_sb (*если включен хотя бы один переключатель: автоматический или полуавтоматический режим*)
THEN avtomat; (* или нажата кнопка сброс то вызывается программа автоматического режима с функцией полуавтоматического режима либо для работы, либо для сброса*)

END_IF (* параметров в автоматическом и полуавтоматическом режиме*)

(* ВКЛЮЧЕНИЕ АВАРИЙНОГО СИГНАЛА ПРЕДОХРАНИТЕЛЯ ПОВОРОТА УЗЛА ВПРЫСКА*)

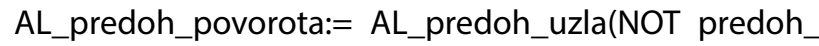
povorota_sq); (*предохранитель поворота - включение аварии когда узел впрыска не намести*)

(*ВКЛЮЧЕНИЕ АВАРИЙНОГО ИНДИКАТОРА НА ПАНЕЛИ ОПЕРАТОРА ${ }^{*}$

AVARIY:= SIGNAL_AL_OR A_temp OR A_predohr_ruch OR (NOT predoh_povorota_sq) OR (NOT ograj_pf_sq) OR (NOT ograj_s_zak_sq) OR (NOT vis_pf_sq)OR ogr_pf_vper_ sq;

( ${ }^{*}$ СЧЁТ КОЛИЧЕСТВА ЗАПРЕСОВОК (ВПрыскОВ) $\left.{ }^{*}\right)$

schet_zapresovok(CU:=vprisk_ya, RESET:= R_schet_zap, $\mathrm{CV}=>$ schet_zapres_OUT);

(*ВЫЗОВ ФУНКЦИОНАЛЬНОГО БЛОКА РЕГУЛИРОВКИ ДАВЛЕНИЯ ПРИ ВПРЫСКЕ ИЛИ ВЫДЕРЖКИ ПОД ДАВЛЕ$\left.\mathrm{HИЕM}^{*}\right)$

(*ВХОДНЫЕ ВЕЛИЧИНЫ*)

stup_davlen.ustavka:=Ust_N_Stup; (*уставка количества ступеней давления*)

stup_davlen.ruch:=ruchnoi_sa; (*режим работы- ручной*)

stup_davlen.nalad:= naladka_sa;(*режим работы- наладочный*)

stup_davlen.avtom:= avtomat_sa;('режим работы- автоматический или полуавтоматический ${ }^{*}$ )

stup_davlen.vprisk:= vprisk_ya; ${ }^{*}$ клапан впрыска включен*)

stup_davlen.stup_davleniy:= stupen_sa; ( ${ }^{*}$ включен режим ступенчатого давления*)

stup_davlen.time_dav:=Time_davlen; (*установка времени выдержки под давлением*)

stup_davlen.kon_vpriska:= kon_vprisk_sq; (*произошло окончание впрыска с переходом на выдержку под давлением*)

(*ВЫХОДНЫЕ ВЕЛИЧИНЫ*)

stup_davlen(ya1=> stupen 1); (*чтение ступеней давления*)

stup_davlen(ya2=> stupen2);

stup_davlen(ya3=> stupen3);

stup_davlen(ya4=> stupen4);

stup_davlen(ya5=> stupen5);

stup_davlen(ya6=> stupen6);

stup_davlen(ya7=> stupen7); 
stup_davlen(ya8=> stupen8);

stup_davlen(ya9=> stupen9);

stup_davlen(schet_OUT_=> N_stup); ( ${ }^{*}$ Выход на экран значения количества ступеней давления*)

Замена системы управления станка вызвала необходимость создания новых электрических принципиальных и монтажных схем. В результате проделанной работы шкаф управления литьевой машины стал значительно компактней. Отпала необходимость использования дополнительного шкафа с советской электроникой «МИКРО 902» который занимал дополнительную полезную площадь.

\section{Выво $\triangle$}

В данной работе кратко изложены этапы создания программы пользователя в среде Codesys v2.3 для программируемых логических контроллеров ПЛК11024.60.Р.М, при выполнении ремонтных работ системы управления литьевой машины ЛПД 500/160. Материал данной статьи нацелен на закрепление теоретических и практических познаний в области средств автоматизации и программирования программируемых логических контроллеров. Данная работа полностью реализована на практике и может быть полезна разработчикам АСУ ТП при ремонте литьевых машин подобного класса.

\section{ЛИТЕРАТУРА}

1. Шишов 0.В. Современные технологии промышленной автоматизации: учебное пособие / 0.В. Шишов. Саранск: Изд-во Мордов. ун-та, 2009.— 280 с.

2. Шишов 0.В. «Программируемые контроллеры в системах промышленной автоматизации» / 0.В. Шишов. — Саранск, 2014. - 448 с.

3. Н.И. Чернявский. «Элементы электрических схем». Ч. 1. / Альбом стандартизированных условно графических и буквенных обозначений: Методические указания по оформлению лабораторных, курсовых и дипломных работ для студентов специальности «Промышленная электроника»,- Тольятти: НГУ, 2007.- 61 c. https://docs.yandex.ru/docs/

4. Программирование программируемых логических контроллеров ОВЕН ПЛК110 и ПЛК160. Руководство пользователя. Версия 1.9. Москва 2010. https:// docs.yandex.ru/docs/view?tm $=16$

5. «Руководство пользователя по программированию ПЛК в среде CoDeSys 2.3». ПК Пролог. Смоленск 2006 г. https://docs.yandex.ru/docs/view?tm=1638

6. Паспорт 70620.002.22.000ПС. Разработано: ОКБ РП0 «Электромеханика» 1989 г.

7. Машина для литья под давлением термопластичных материалов. Модель ЛПД-500/160. Руководство по эксплуатации 70620.002.00.000Рэ. Савеловское производственное объединение «Прогресс» 1989 г.

8. Система управления PLAS-S руководство оператора 501.00050-01 <34 <01 <1991 г.

9. Официальный сайт компании «Овен» [Электронный ресурс]. — Режим доступа: www.owen.ru.

10. Оссвальд Т., Турнг Л.-Ш., Грэманн П. Дж., «Литье пластмасс под давлением» под ред. Э.Л. Калинчева, ISBN: 5-93913-067-4, Изд-в0: «Ц0П Профессия» 2005-712 c. https://plastinfo.ru

(с) Русаков Максим Геннадьевич ( е10е00@mail.ru),

Евдокимов Александр Сергеевич (evd15@list.ru ), Занкин Александр Иванович (zankin_ai@mail.ru).

Журнал «Современная наука: актуальные проблемы теории и практики» 